Cite as: Stephane H Maes, (2020), "Strong CP Violation Tamed in The Presence of Gravity", viXra:2007.0025v1, https://vixra.org/pdf/2007.0025v1.pdf, https://shmaesphysics.wordpress.com/2020/06/23/ strong-cp-violation-tamed-in-the-presence-of-gravity/, June 21, 2020.

\title{
Strong CP Violation Tamed in The Presence of Gravity
}

\author{
Stephane H. Maes ${ }^{1}$
}

June 23, 2020

\begin{abstract}
:
In a multi-fold universe, gravity emerges from entanglement through the multi-fold mechanisms. As a result, gravity-like effects appear in between entangled particles that they be real or virtual. Long range, massless gravity results from entanglement of massless virtual particles. Entanglement of massive virtual particles leads to massive gravity contributions at very smalls scales. Multi-folds mechanisms also result into a spacetime that is discrete, with a random walk fractal structure and non-commutative geometry that is Lorentz invariant and where spacetime nodes and particles can be modeled with microscopic black holes. All these recover General relativity at large scales and semi-classical model remain valid till smaller scale than usually expected. Gravity can therefore be added to the Standard Model. This can contribute to resolving several open issues with the Standard Model.

The strong CP violation problem is one of these issues: $Q C D$ predicts $C P$ violation, yet no $C P$ violation has ever been observed involving the strong interaction (when it occurs, it is for the weak interaction). In this paper we show that when adding gravity to the Standard Model, in a multi-fold universe, gravity allows the mass of the up quark to be smaller (close to, or equal to zero). This symmetry, or quasi symmetry, is a way to eliminate the CP violation contributions in QCD, therefore resolving the problem. It argues for evolving the Standard Model to add gravity, if non negligible at very small scales. No New Physics are introduced as new particles, which could also explain why axions have never been observed, and we may have to remove them as candidates to explain dark matter.
\end{abstract}

\section{Introduction}

The new preprint [1] proposes contributions to several open problems in physics like the reconciliation of General Relativity (GR) with Quantum Physics, explaining the origin of gravity proposed as emerging from quantum (EPREinstein Podolsky Rosen) entanglement between particles, detailing contributions to dark matter and dark energy and explaining other Standard Model mysteries without requiring New Physics beyond the Standard Model other than the addition of gravity to the Standard Model Lagrangian. All this is achieved in a multi-fold universe that may well model our real universe, which remains to be validated.

With the proposed model of [1], spacetime and Physics are modeled from Planck scales to quantum and macroscopic scales and semi classical approaches appear valid till very small scales. In [1], it is argued that spacetime is discrete, with a random walk-based fractal structure, fractional and noncommutative at, and above Planck scales (with a 2-D behavior and Lorentz invariance preserved by random walks till the early moments of the universe). Spacetime results from past random walks of particles. Spacetime locations and particles can be modeled as microscopic black holes (Schwarzschild for photons and spacetime coordinates, and metrics between Reisner Nordstrom [2] and Kerr Newman [3] for massive and possibly charged particles - the latter being possibly extremal). Although surprising, [1] recovers results consistent with other like [4], while also being able to justify the initial assumptions of black holes from the gravity or entanglement model. The resulting gravity model recovers General Relativity at larger scale, as a 4-D process, with massless gravity, but also with massive gravity components

\footnotetext{
${ }^{1}$ shmaes.physics@gmail.com
} 
at very small scale that make gravity significant these scales. Semi-classical models also work well till way smaller scales that usually expected.

In this paper, we remain at a high level of discussion of the analysis and references are generic for the subjects. It makes the points accessible to a wider audience and keeps the door open to further papers or discussions devoted to details of interest. Yet, it requires the reader to review [1], as we do not revisit here all the details of the multifold mechanism or reconstruction of spacetime. More targeted references for all the material discussed here are compiled in [1].

\section{2. $\mathrm{SM}_{\mathrm{G}}$ : The Standard Model with Multi-Fold Gravity}

[1] proposes that, in a multi-fold universe, the Lagrangian is complemented by terms associated to gravity and entanglement (in the form of the sum of the attractive effective potentials) [1].

$$
\mathscr{L}_{\text {total }}=\mathscr{L}_{S M}+\mathscr{L}_{\text {Gravity-with }-M F}+\mathscr{L}_{\text {EPR }}+\mathscr{L}_{\text {Others } \ldots}
$$

The effect of gravity can be seen through the attractive potential contributions of all the energy sources, static or moving (hence modeled by the energy momentum 4-vector). It can also been seen as expressing the Standard Model Lagrangian in curved spacetime (semi-classical point of view), now considered valid till small scales.

EPR entanglement is not believed to often play a significant role, except in dark matter use cases [5].

The last term is all other "New Physics" terms and we will consider it to be null.

With (1), the mass generation terms now involves the gravity effective potential described in [1]:

$$
\begin{aligned}
\mathscr{L}_{\text {int }} & \propto C_{1}(\text { vertex }) \\
& +m_{\text {acquired }}\left(1+\frac{V_{\text {eff }}}{m_{\text {acquired }}}\right)\left(C_{2}(\text { lepton } / \text { quark })\right. \\
& +C C)
\end{aligned}
$$

Where, the $C_{1}$ () designates the vertex contribution that represents the interaction with the Higgs/QCD Vacuum (and/or Quark Gluon condensate) with chirality flip and $C_{2}($ ) represents the contributions of the right-handed and left-handed leptons or quarks and anti-particles.

\section{The Strong CP Violation Problem}

Overviews of the Strong-CP violation problems can be found in [6] with a popular science discussion in [7]. Quantum Chromodynamic (QCD), which models with high accuracy string interactions among quarks and gluons, as well as between nuclei, predicts a CP violation term.

Yet there have never been any observation of $\mathrm{CP}$ violations involving the strong interaction. $\mathrm{CP}$ violations have been observed but they involve the weak interaction [8]. This puzzle is known as the Strong CP violation problem.

[9] discusses in great details the violation in QCD and how to explain its apparent nonexistence. As is often the case, introducing symmetries and symmetry breaking helps when these kind of problems are met in Physics. So, 
one of the popular hypothesis relies on the introduction of a new symmetry with a scalar field and a particle: the axion [10].

Unfortunately, the axions is not expected to interact much with rest of the world and it has, so far, never been observed (in experiences where axions are expected to interact with a strong magnetic field and converts to photons). So we do not know if the Peccei-Quinn symmetry exists [12]. The flip side of this, is that the axion became an ideal candidate to explain dark matter [11]. Yet, [1,5] suggest an entanglement-based explanation for dark matter (at least a portion of the effects).

If axions do not exists, axiogenesis, proposed to explain why the universe has matter, also collapses [13,14].

\section{Gravity and The Quark Mass: No More Strong CP Violation Problem}

The solution proposed by [1] is straightforward. Gravity is no more negligible at the strong interaction scales, mostly because of the massive gravity contributions. In the case of the up quark (lightest quark), the mass term, conventionally assumed to represent the mass quark, can actually originate (in part) from $V_{\text {eff, }}$ the gravity effective potential contributions. Therefore, $\mathrm{m}_{\mathrm{u}}$, the mass of the up quark, can be significantly reduced and correspond to a quasi-zero rest mass. If that is the case, the offending terms in QCD, responsible for CP violation, disappears [9].

The mystery is solved. It is achieved within the context of the Standard Model with Gravity, in a multi-fold universe, and without the need of New Physics.

Also, this analysis is for a Multi-fold universe as in [1]. [1] details arguments and ways to check its relationship with the real universe. Besides properties that can be experimentally verified (in the future because of the macroscopic weakness of gravity and gravity like effects for entangled systems), [1] shows how the multi-fold mechanisms and behaviors are in many aspects in today's conventional physics, that, at times, anticipates the behaviors modeled of a multi-fold universe. In addition, [1] explains many results obtained in gravity, quantum mechanics, General Relativity, superstring theory, Loop Quantum Gravity and the AdS/CFT correspondence conjecture. All these works attempt to come up with models for the real universe. It is at least a good sign that [1] may provide an interesting model of the real universe.

Other theories showing that gravity is relevant at the level of the standard model, can repeat the $m_{u}$, the mass of the up quark, reduction argument, even with no relation to multi-fold universe and mechanisms or to gravity emergence from entanglement. So our model here is generic: if we add gravity to Standard Model with a model keeping it non negligible at the Standard Model scales, then the CP violating term in QCD disappears.

If our model here is not validated, by experience, it would not invalidate the multi-fold mechanism and the proposal that gravity emerges from entanglement as detailed in [1]. The analysis builds on [1], as a consequence of it, but it is not a condition for validation of multi-fold universes.

Note the recent possible sightings of axions (or at least anomalous events) reported in [17]. Considering the analysis below, it is not too likely that these are due to axions. It is important to understand that our approach does not forbid axions and the Peccei-Quinn symmetry. We only stated that it may not be needed in the context of explaining the strong CP violation problem. Even the Peccei-Quinn symmetry exists, it can combine with our proposal (or render our argument moot), yet without implying that our proposal does not also occur in terms of $m_{u}$, the mass of the up quark.

\section{Conclusions}


We believe that [1] makes a compelling case for the consistency of its multi-fold proposal. The present paper shows how the mechanisms of multi-fold universes can help address the strong CP violation problem.

We explain how the addition of gravity to the QCD Lagrangian (and Standard Model), can eliminate the term responsible for $\mathrm{CP}$ violation in $\mathrm{QCD}$, therefore resolving the strong $\mathrm{CP}$ violation problem.

The model works for multi-fold universe as well as in any situation where gravity is non negligible and added to the Standard Model.

This, along with similar results in [1] and [15,16], make a strong case for more seriously considering the implications of adding gravity to the Standard Model to obtain $\mathrm{SM}_{\mathrm{G}}$, as a way to addressing open issues and offer better alternatives to New Physics speculations. This goes hand in hand with recognizing that this also implies the need to seriously consider that gravity may not always be negligible at the Standard Model scales as proposed in [1].

References: (most references come from popular science to make the discussion more approachable)

[1]: Stephane H. Maes, (2020) "Quantum Gravity Emergence from Entanglement in a Multi-Fold

Universe", viXra:2006.0088v1, (June 9, 2020).

[2]: https://en.wikipedia.org/wiki/Reissner\%E2\%80\%93Nordstr\%C3\%B6m metric

[3]: https://en.wikipedia.org/wiki/Kerr-Newman metric

[4]: Burinskii, Alexander, (2008), "The Dirac-Kerr-Newman electron", arXiv:0507109v4

[5]: Stephane H Maes, (2020), "Explaining Dark Matter Without New Physics?",

viXra:2007.0006v1, https://vixra.org/pdf/2007.0006v1.pdf or

https://shmaesphysics.wordpress.com/2020/06/19/explaining-dark-energy-small-cosmological-constant-andinflation-without-new-physics/, June 21, 2020.

[6]: https://en.wikipedia.org/wiki/Strong CP problem

[7]: https://www.forbes.com/sites/startswithabang/2019/11/19/the-strong-cp-problem-is-the-most-underratedpuzzle-in-all-of-physics/

[8]: https://en.wikipedia.org/wiki/CP violation

[9]: I. I. Bigi and A. I. Sanda, (2009), "CP Violation", Cambridge University Press

[10]: https://en.wikipedia.org/wiki/Axion

[11]: https://www.quantamagazine.org/why-dark-matter-might-be-axions-20191127/

[12]: R. D. Peccei and Helen R. Quinn, (1977), "CP Conservation in the Presence of Pseudoparticles", Phys. Rev. Lett. 38, 1440

[13]: https://www.quantamagazine.org/axions-would-solve-another-major-problem-in-physics-20200317/

[14]: Raymond T. Co, Keisuke Harigaya, (2019), "Axiogenesis", arXiv:1910.02080v2.

[15]: Stephane H Maes, (2020), "Gravity Induced Anomalies Smearing in Standard Model so that Protons May

Never Decay, Except in Black Holes ", viXra:2006.0128v1, https://shmaesphysics.wordpress.com/2020/06/12/protons-may-never-decay-except-in-black-holes/, June 13, 2020.

[16]: Stephane H Maes, (2020), "Right-handed neutrinos? Mass? Ask Gravity", viXra:2007.0018v1, https://shmaesphysics.wordpress.com/2020/06/21/right-handed-neutrinos-ask-gravity/, June 21, 2020.

[17]: E. Aprile, et al., (2020), "Observation of Excess Electronic Recoil Events in XENON1T", arXiv:2006.09721v1 